\title{
Global Saturated Regulator with Variable Gains for Robot Manipulators
}

\author{
Brandon Sánchez-García* ${ }^{1}$, Fernando Reyes-Cortés ${ }^{2}$, Basil M. Al-Hadithi ${ }^{3,4}$ and Olga Félix-Beltrán ${ }^{5}$ \\ 1,2,5 Facultad de Ciencias de la Electrónica, Benémerita Universidad Autónoma de Puebla, Puebla, México. \\ ${ }^{3}$ Centre for Automation and Robotics UPM-CSIC, Intelligent Control Group, Universidad Politécnica de Madrid, Spain. \\ ${ }^{4}$ Department of Electrical, Electronics, Control Engineering and Applied Physics, Higher Technical School of Industrial \\ Design and Engineering, Universidad Politécnica de Madrid, Madrid, Spain. \\ Email: ${ }^{1}$ brandon.sanchez@alumno.buap.mx \\ *Corresponding Author
}

\begin{abstract}
In this paper we propose a set of saturated controllers with variable gains to solve the regulation problem for robot manipulators in joint space. These control schemes deliver torques inside prescribed limits of servomotors. The gamma of variable gains is formed by continuous, smooth, and differentiable functions of the joint position error and velocity of manipulator. A strict Lyapunov function is proposed to demonstrate globally asymptotic stability of the close-loop equilibrium point. Finally, the functionality and performance of the proposal is illustrated via simulation results and comparative analysis against Proportional-Derivative (PD) control scheme on a two-degrees-freedom direct-drive robot manipulator.
\end{abstract}

Keywords-Saturated controllers, global asymptotic stability, variable gains, self-tuning, robot manipulator.

\section{INTRODUCTION}

The robot manipulators are mechatronic systems that solve problems where the human is limited. This can be seen in the different activities of industrialized countries such as, increased production, manufacturing engineering and robotic medicine. Other field is exploration, today the robot manipulators are needed to study different ecosystems. Thus, scientists obtain many new information about the flora and fain. The trend of exploration aims to Mars and other planets; NASA has said that it is the biggest challenge for robotics. Nonetheless, for a robot to carry out complex activities, it is necessary to implement a high-performance control. For this reason, the scientific community in robotic has focused on design controllers, such as these features is contained [1].

To indicate a certain robot task is necessary to solve the trajectory problem. On the other hand, the regulation problem in joint space is a case of the trajectory problem, which consists of designing a regulator such that, carry to joint positions from any initial conditions to certain desired positions of robot manipulator. The solutions can be extrapolated to other motion control application, such as point to point control or cartesian control. However, one of the practical problems is saturation; due to the servomotors of the robot manipulator do not have an unlimited source of torque. This problem generates unmodeled mechanics on the robot manipulator. The classical PD and ProportionalIntegral-Derivative (PID) regulator have the disadvantage of applying unlimited torque; hence, the servomotors can work in the saturation zone [2]. In this case, the applied torque by the actuators to the manipulator joints will be different from that demanded by the controller. Furthermore, saturation of the actuators deteriorates the control performance and leads to thermal and mechanical failure [5].

These control schemes have been modified to avoid this problem using different perspectives. In [3] on a SaturatedProportional scheme is proposed; through hyperbolic tangent function, the control actions have been saturated. A Saturated Proportional-Saturated Derivative (SP-SD) control is presented and implemented on a two-degrees-of-freedom robot in [4]. We highlight [5] where a simple controller is shown, by continuous functions, this control delivers bounded torques; in this sense, a SP-SD adaptive control is implemented on a robot system. A lineal PD feedback is presented in [6], which the integral action is driven by a nonlinear function of the joint position error of the robot manipulator. Years later, a new saturated nonlinear PID global regulator is proposed in [7] such as, bounded torques are generated. A saturated nonlinear PID regulator for industrial robot manipulators is presented in [8] as alternative to solve the saturation problem.

The energy shaping methodology is another perspective for dynamic control of robot manipulators, which is presented in by Takegaki and Arimoto [9]; from this technique, many papers have been proposed. In [10] the designed controller is capable to apply bounded torque by a known trigonometric function for saturation of control actions. Also, a group of saturated controllers is shown in [11]; these schemes are formed by a combination of functions such as, the limited control actions is generated. The gradient of artificial potential energy is represented by square root-type to design a new regulator with bounded control actions, the implementation and experimental results are presented in [12]. The contribution [13] shows as, the energy shaping methodology allows generating families of control schemes; these controllers have different qualitive proprieties to enhance feedback control of robot, global asymptotic stability, and experimental results on a direct-drive-robot.

The main disadvantage is, the previous control schemes have constant gains, the control-gains-tune is complicated, and the empirical method by the user is needed. A wrong control-gains-tune causes a low performance. In the literature, there are different papers where this problem is approached. The fuzzy logic is the most used. In [14] a fuzzy 
self-tuning procedure is implemented for a PD control with gravity compensation. A motion control scheme based on a gain scheduling fuzzy self-tuning structure for robot manipulator is presented in [15]. Later, a new PID is presented in [16] which is based on a fuzzy system for tuning gains at the same time proving the semiglobal asymptotic stability via Lyapunov's direct method. In recent works, we highlight [17] where a new robust controller is developed for robot manipulators based on an integrating between a novel fuzzy self-tuning PID-nonsingular fast terminal sliding mode control; results is illustrated on a PUMA560 robot.

From the context of the neural networks, various authors have approached the control-gains-tune problem. A new method is presented in [18] for design of multivariable selftuning PID based on quasi-diagonal recurrent wavelet neural network, getting better learning ability, generalization of wavelet transforms, dynamic mapping and quickly convergence. In [19] the neural network self-tuning PID control is presented, where the proportional, derivative, and integral gains are self-tuning on-line by neural network output which is obtained due to the error value on the desired output of the system under control. An approach of the selftuning control with neural network for robot manipulator in an object balancing task is proposed in [20]; the neural network learns and self-calibrate by some repeating trail movements until the virtual feedback enable the robot adopts recalibrating parameters to stabilize the rolling task. Afterwards, in [21] a radial basis function neuronal networks are used to estimate uncertainties and determinate PID gains.

Other interesting propose is Particle Swarm Optimization (PSO) technique. This algorithm is inspired by social behavior of animals like ants and birds. This scheme is an optimization problem, which contains a set of swarm solutions called particles that has a position in a multidimensional search space, which contains all possible solutions within optimization problem. The PSO searches for the optimum solution among these possible solutions. In this sense, in [22] PSO-based-tuning for the control gains is proposed for a hyperbolic inverse regulator with global asymptotic stability and implemented on an experimental direct-drive-robot manipulator.

Finally, the modification of the controller gains by continuous functions and then tune it, is an optimal solution. In [23] is presented a modification of PD regulator such as the proportional and derivative gains can be nonlinear functions of the robot states at the same time stability analysis. Later, the same idea is presented in [24] with a different nonlinear PD with experimental results on open architecture platform for robot manipulators. Other PD controller is presented in [25] with the variable gains concept; the tuning and simulation is illustrated for tracking control applications. A quasi-variable PID and asymptotic stability analysis is presented in [26] where the control gains are nonlinear functions of the joint position error. The work presented in [27], also a PD with variable gains is proposed defining self-tuning rules and implementation on a robot manipulator. Years later, an unbounded controller with variable gains through a hyperbolic sine function, asymptotic stability demonstration and experimental results is presented in [28]. On the other hand, a saturated controller with derivative variable gain is proposed in [29] with global asymptotic stability using strict Lyapunov function and experimental results on a three degrees of freedom directdrive-robot manipulator.

Nonetheless, the previous papers do not give a solution to saturation problem and self-tuning control-gains by means of saturated controllers with global asymptotic stability and a gamma of proportional and derivative variable gains, structured by continuous functions of the joint position error and velocity of robot. The self-tuning is realized by a design law using the maximum torque delivered by each servomotor of the robot manipulators; hence, the designer to perform an optimal, practical, and simple tuning. Furthermore, the saturated controllers allow the servomotors are working within the prescribed limits then, the saturation problem is solved. Also, the mathematical structure provides driving the position error to zero and, the same time, damping energy of the derivative term is obtained. On a practical level, the robot has response without oscillation in the transitory regime and soft convergence to permanent regime.

An important contribution of this paper is the global asymptotic stability demonstration of the close-loop equilibrium point, formed by robot dynamics and saturated controllers with variable gains, via Lyapunov's direct method. A strict Lyapunov function is proposed which satisfies being a positive definite function and its derivative is negative definite function.

For the propose of illustrating the functionality and performance, the controllers are implemented on a two degrees freedom direct-drive-robot via simulation and comparing the performance with a PD scheme control. The control-gains-tune is realized manually.

The organization of this paper is as follow: Section 2 focuses on the presentation of the robot dynamics, the proprieties that satisfies and useful propriety for integrable functions, which is demonstrated. The gamma of variable gains is presented in Section 3. Section 4 presents the saturated controllers with variable gains, the regulation problem and shows the global asymptotic stability analysis by strict Lyapunov function. Section 5 describes self-tuning law for variable gains. Section 6 presents the simulation results, performance, and comparative analysis against PD control scheme. Finally, conclusions are presented in Section 7.

\section{ROBOT DYNAMICS}

Consider the nonlinear dynamics of a n-degrees-offreedom rigid robot manipulator with revolute joints:

$$
M(\boldsymbol{q}) \ddot{\boldsymbol{q}}+C(\boldsymbol{q}, \dot{\boldsymbol{q}}) \dot{\boldsymbol{q}}+\boldsymbol{g}(\boldsymbol{q})+B \dot{\boldsymbol{q}}=\boldsymbol{\tau}
$$

where $\boldsymbol{q}, \dot{\boldsymbol{q}}, \ddot{\boldsymbol{q}} \in \mathbb{R}^{n}$ are the position, velocity and joint acceleration, respectively; $\boldsymbol{\tau} \in \mathbb{R}^{n}$ is the vector of inputs torques, $M(\boldsymbol{q}) \in \mathbb{R}^{n \times n}$ is the symmetric positive definite manipulator inertia matrix, $C(\boldsymbol{q}, \dot{\boldsymbol{q}}) \in \mathbb{R}^{n \times n}$ is the matrix of centripetal and Coriolis torques, $\boldsymbol{g}(\boldsymbol{q}) \in \mathbb{R}^{n}$ is the vector of gravitational torques obtained as gradient of the robot potential energy due to gravity, and $B \in \mathbb{R}^{n \times n}$ is the positive definite diagonal matrix for the viscous friction 
torques. Although the equation motion (1) is complex and satisfies different proprieties. For the proposed controller, the following important proprieties are used:

\section{Property 2.1}

The matrix of inertia $M(\boldsymbol{q}) \in \mathbb{R}^{n \times n}$ is symmetric and positive definite, it satisfies:

$$
\begin{aligned}
& M(\boldsymbol{q})>0 ; M(\boldsymbol{q})=M(\boldsymbol{q})^{T} \\
& M(\boldsymbol{q})^{-1}>0 ; \boldsymbol{x}^{T} M(\boldsymbol{q}) \boldsymbol{x}>0
\end{aligned}
$$

for all $\boldsymbol{x} \in \mathbb{R}^{n}$.

\section{Property 2.2}

If $\dot{\boldsymbol{q}}=\mathbf{0}$, then the centrifugal and Coriolis torques matrix satisfies $C(\boldsymbol{q}, \mathbf{0})=0 \in \mathbb{R}^{n \times n} ;$ for all $\boldsymbol{q} \in \mathbb{R}^{n}$.

\section{Property 2.3}

The time derivative of the inertia matrix $\dot{M}(\boldsymbol{q})$ is symmetric also, and satisfies:

$$
\dot{M}(\boldsymbol{q})=C(\boldsymbol{q}, \dot{\boldsymbol{q}})^{T}+C(\boldsymbol{q}, \dot{\boldsymbol{q}})
$$

\section{Property 2.4}

The centrifugal Coriolis matrix and the derivative of the inertia matrix satisfy:

$$
\frac{1}{2} \dot{\boldsymbol{q}}^{T}[\dot{M}(\boldsymbol{q})+2 C(\boldsymbol{q}, \dot{\boldsymbol{q}})] \dot{\boldsymbol{q}}=0
$$

for all $\boldsymbol{q}, \dot{\boldsymbol{q}} \in \mathbb{R}^{n}$.

\section{Property 2.5}

There exists a constant $k_{c}$ such that:

$$
\|C(\boldsymbol{q}, \dot{\boldsymbol{q}}) \dot{\boldsymbol{q}}\| \leq k_{\boldsymbol{c}}\|\dot{\boldsymbol{q}}\|^{2}
$$

for all $\boldsymbol{q}, \dot{\boldsymbol{q}} \in \mathbb{R}^{n}$.

\section{Property 2.6}

If positive constants $k_{p u}$ and $k_{p l}$ exist such that, represent upper and lower bounds of certain continuously integrable functions such that satisfies:

$$
\begin{aligned}
& k_{p u} \geq k_{p i}\left(\tilde{q}_{i}, \dot{q}_{i}\right) \geq k_{p l} ; \forall \tilde{q}_{i}, \dot{q}_{i} \in \mathbb{R}, \text { then: } \\
& \frac{1}{2} k_{p l}\|\widetilde{\boldsymbol{q}}\|^{2} \leq \int_{\mathbf{0}}^{\widetilde{\boldsymbol{q}}} \boldsymbol{\sigma}^{T} K_{p}(\boldsymbol{\sigma}, \dot{\boldsymbol{q}}) d \boldsymbol{\sigma} \leq \frac{1}{2} k_{p u}\|\widetilde{\boldsymbol{q}}\|
\end{aligned}
$$

Proof 1 Using integration by parts method:

$$
\begin{aligned}
\int_{\mathbf{0}}^{\widetilde{\boldsymbol{q}}} \boldsymbol{\sigma}^{T} K_{p}(\boldsymbol{\sigma}, \dot{\boldsymbol{q}}) d \boldsymbol{\sigma} & =\widetilde{\boldsymbol{q}}^{T} K_{p}(\widetilde{\boldsymbol{q}}, \dot{\boldsymbol{q}}) \widetilde{\boldsymbol{q}} \\
& -\boldsymbol{\sigma}^{T}(0) K_{p}(\boldsymbol{\sigma}(0), \dot{\boldsymbol{q}}) \boldsymbol{\sigma}(0) \\
& -\int_{\mathbf{0}}^{\widetilde{\boldsymbol{q}}} \boldsymbol{\sigma}^{T} K_{p}(\boldsymbol{\sigma}, \dot{\boldsymbol{q}}) d \boldsymbol{\sigma} \\
& -\int_{\mathbf{0}}^{\widetilde{\boldsymbol{q}}} \boldsymbol{\sigma}^{T} \dot{K}_{p}(\boldsymbol{\sigma}, \dot{\boldsymbol{q}}) d \boldsymbol{\sigma}
\end{aligned}
$$

In this context, we have:

$$
\begin{aligned}
\int_{\mathbf{0}}^{\widetilde{\boldsymbol{q}}} \boldsymbol{\sigma}^{T} K_{p}(\boldsymbol{\sigma}, \dot{\boldsymbol{q}}) d \boldsymbol{\sigma} & =\widetilde{\boldsymbol{q}}^{T} K_{p}(\widetilde{\boldsymbol{q}}, \dot{\boldsymbol{q}}) \widetilde{\boldsymbol{q}} \\
& -\int_{\mathbf{0}}^{\widetilde{\boldsymbol{q}}} \boldsymbol{\sigma}^{T} K_{p}(\boldsymbol{\sigma}, \dot{\boldsymbol{q}}) d \boldsymbol{\sigma} \\
& -\int_{\mathbf{0}}^{\widetilde{\boldsymbol{q}}} \boldsymbol{\sigma}^{T} \dot{K}_{p}(\boldsymbol{\sigma}, \dot{\boldsymbol{q}}) d \boldsymbol{\sigma}
\end{aligned}
$$

$$
\begin{aligned}
& \int_{\mathbf{0}}^{\widetilde{\boldsymbol{q}}} \boldsymbol{\sigma}^{T} K_{p}(\boldsymbol{\sigma}, \dot{\boldsymbol{q}}) d \boldsymbol{\sigma} \leq \widetilde{\boldsymbol{q}}^{T} K_{p}(\widetilde{\boldsymbol{q}}, \dot{\boldsymbol{q}}) \widetilde{\boldsymbol{q}} \\
&-\int_{\mathbf{0}}^{\widetilde{\boldsymbol{q}}} \boldsymbol{\sigma}^{T} K_{p}(\boldsymbol{\sigma}, \dot{\boldsymbol{q}}) d \boldsymbol{\sigma} \\
& 2 \int_{\mathbf{0}}^{\widetilde{\boldsymbol{q}}} \boldsymbol{\sigma}^{T} K_{p}(\boldsymbol{\sigma}, \dot{\boldsymbol{q}}) d \boldsymbol{\sigma} \leq \widetilde{\boldsymbol{q}}^{T} K_{p}(\widetilde{\boldsymbol{q}}, \dot{\boldsymbol{q}}) \widetilde{\boldsymbol{q}}
\end{aligned}
$$

Note that:

$$
\begin{aligned}
& \widetilde{\boldsymbol{q}}^{T} K_{p}(\widetilde{\boldsymbol{q}}, \dot{\boldsymbol{q}}) \widetilde{\boldsymbol{q}} \leq\left|\widetilde{\boldsymbol{q}}^{T} K_{p}(\widetilde{\boldsymbol{q}}, \dot{\boldsymbol{q}}) \widetilde{\boldsymbol{q}}\right| \\
& \widetilde{\boldsymbol{q}}^{T} K_{p}(\widetilde{\boldsymbol{q}}, \dot{\boldsymbol{q}}) \widetilde{\boldsymbol{q}} \leq\left\|\widetilde{\boldsymbol{q}}^{T}\right\|\left\|K_{p}(\widetilde{\boldsymbol{q}}, \dot{\boldsymbol{q}}) \widetilde{\boldsymbol{q}}\right\| \\
& \widetilde{\boldsymbol{q}}^{T} K_{p}(\widetilde{\boldsymbol{q}}, \dot{\boldsymbol{q}}) \widetilde{\boldsymbol{q}} \leq \lambda_{\operatorname{Max}}\left\{K_{p}(\widetilde{\boldsymbol{q}}, \dot{\boldsymbol{q}})\right\}\|\widetilde{\boldsymbol{q}}\|^{2} \\
& \widetilde{\boldsymbol{q}}^{T} K_{p}(\widetilde{\boldsymbol{q}}, \dot{\boldsymbol{q}}) \widetilde{\boldsymbol{q}} \leq \lambda_{\operatorname{Max}}\left\{K_{p}(\widetilde{\boldsymbol{q}}, \dot{\boldsymbol{q}})\right\}\|\widetilde{\boldsymbol{q}}\|^{2} \\
& \widetilde{\boldsymbol{q}}^{T} K_{p}(\widetilde{\boldsymbol{q}}, \dot{\boldsymbol{q}}) \widetilde{\boldsymbol{q}} \leq k_{p u}\|\widetilde{\boldsymbol{q}}\|^{2}
\end{aligned}
$$

Under expression (9) and (10) the following inequality is satisfied:

$$
\int_{\mathbf{0}}^{\widetilde{\boldsymbol{q}}} \boldsymbol{\sigma}^{T} K_{p}(\boldsymbol{\sigma}, \dot{\boldsymbol{q}}) d \boldsymbol{\sigma} \leq \frac{1}{2} k_{p u}\|\widetilde{\boldsymbol{q}}\|^{2}
$$

thus, since context on expression (8):

$$
\begin{aligned}
\int_{\mathbf{0}}^{\widetilde{\boldsymbol{q}}} \boldsymbol{\sigma}^{T} K_{p}(\boldsymbol{\sigma}, \dot{\boldsymbol{q}}) d \boldsymbol{\sigma} & =\widetilde{\boldsymbol{q}}^{T} K_{p}(\widetilde{\boldsymbol{q}}, \dot{\boldsymbol{q}}) \widetilde{\boldsymbol{q}} \\
& -\int_{\mathbf{0}}^{\widetilde{\boldsymbol{q}}} \boldsymbol{\sigma}^{T} K_{p}(\boldsymbol{\sigma}, \dot{\boldsymbol{q}}) d \boldsymbol{\sigma} \\
& -\int_{\mathbf{0}}^{\widetilde{\boldsymbol{q}}} \boldsymbol{\sigma}^{T} \dot{K}_{p}(\boldsymbol{\sigma}, \dot{\boldsymbol{q}}) d \boldsymbol{\sigma}
\end{aligned}
$$

$$
\begin{aligned}
2 \int_{\mathbf{0}}^{\widetilde{\boldsymbol{q}}} \boldsymbol{\sigma}^{T} K_{p}(\boldsymbol{\sigma}, \dot{\boldsymbol{q}}) d \boldsymbol{\sigma} & =\widetilde{\boldsymbol{q}}^{T} K_{p}(\widetilde{\boldsymbol{q}}, \dot{\boldsymbol{q}}) \widetilde{\boldsymbol{q}} \\
+ & \int_{\widetilde{\boldsymbol{q}}}^{0} \boldsymbol{\sigma}^{T} \dot{K}_{p}(\boldsymbol{\sigma}, \dot{\boldsymbol{q}}) d \boldsymbol{\sigma}
\end{aligned}
$$

Then

$$
\begin{aligned}
2 \int_{\mathbf{0}}^{\widetilde{\boldsymbol{q}}} \boldsymbol{\sigma}^{T} K_{p}(\boldsymbol{\sigma}, \dot{\boldsymbol{q}}) & d \boldsymbol{\sigma} \geq \widetilde{\boldsymbol{q}}^{T} K_{p}(\widetilde{\boldsymbol{q}}, \dot{\boldsymbol{q}}) \widetilde{\boldsymbol{q}} \\
\geq & \frac{1}{2} \lambda_{\min }\left\{K_{p}(\widetilde{\boldsymbol{q}}, \dot{\boldsymbol{q}})\right\}\|\widetilde{\boldsymbol{q}}\|^{2} \\
& \geq \frac{1}{2} k_{p l}\|\widetilde{\boldsymbol{q}}\|^{2}
\end{aligned}
$$

therefore, the expression (6) is satisfied. 


\section{GAMMA OF VARIABLE GAINS}

The control scheme proposed, is structured by control gains modification. The proportional $K_{p}(\widetilde{\boldsymbol{q}}, \dot{\boldsymbol{q}}) \in \mathbb{R}^{n \times n}$ and derivative $K_{v}(\widetilde{\boldsymbol{q}}, \dot{\boldsymbol{q}}) \in \mathbb{R}^{n \times n}$ are definite positive diagonal matrices, whose entries are continuous functions of the joint position error $\widetilde{\boldsymbol{q}}=\boldsymbol{q}_{\boldsymbol{d}}-\boldsymbol{q} \in \mathbb{R}^{n}$ and joint velocity $\dot{\boldsymbol{q}} \in \mathbb{R}^{n}$ of the robot manipulator. Being $\boldsymbol{q}_{\boldsymbol{d}} \in \mathbb{R}^{n}$ constant desired positions. In the expressions (15) and (16) we present the gamma of variable gains.

$$
\begin{aligned}
K_{p}(\widetilde{\boldsymbol{q}}, \dot{\boldsymbol{q}}) & =\left[\begin{array}{ccc}
k_{p 1} \cos ^{2}\left(f_{p}\left(\tilde{q}_{1}, \dot{q}_{1}\right)\right) & \cdots & 0 \\
\vdots & \ddots & \vdots \\
0 & \cdots & k_{p n} \cos ^{2}\left(f_{p}\left(\tilde{q}_{n}, \dot{q}_{n}\right)\right)
\end{array}\right] \\
K_{v}(\widetilde{\boldsymbol{q}}, \dot{\boldsymbol{q}}) & =\left[\begin{array}{ccc}
k_{v 1} \cos ^{2}\left(f_{p}\left(\tilde{q}_{1}, \dot{q}_{1}\right)\right) & \cdots & 0 \\
\vdots & \ddots & \vdots \\
0 & \cdots & k_{v n} \cos ^{2}\left(f_{p}\left(\tilde{q}_{n}, \dot{q}_{n}\right)\right)
\end{array}\right]
\end{aligned}
$$

Since $i=1,2, \ldots, n$ : being $i^{\text {th }}$ joint of robot manipulator; $f_{p}\left(\tilde{q}_{i}, \dot{q}_{i}\right)$ and $f_{v}\left(\tilde{q}_{i}, \dot{q}_{i}\right)$ are continuous, differentiable, and smooth functions with upper and lower bounds. In other words $f_{p}\left(\tilde{q}_{i}, \dot{q}_{i}\right)$ and $f_{v}\left(\tilde{q}_{i}, \dot{q}_{i}\right) \in \mathbb{R}^{2} \rightarrow \mathbb{R}$, such that satisfy $v_{p l} \leq f_{p}\left(\tilde{q}_{i}, \dot{q}_{i}\right) \leq v_{p u}$ and $v_{v l} \leq f_{v}\left(\tilde{q}_{i}, \dot{q}_{i}\right) \leq v_{v u} ; \forall \tilde{q}, \dot{q} \in$ $\mathbb{R}$. The coefficients $k_{p i}>0$ and $k_{v i}>0$. Hence, the proportional and derivative gains are positive definite matrices: $\quad K_{p}(\widetilde{\boldsymbol{q}}, \dot{\boldsymbol{q}})>0, \quad K_{v}(\widetilde{\boldsymbol{q}}, \dot{\boldsymbol{q}})>0$. For measure functionality of variable gains, we enlist some cases as following:

$$
\begin{gathered}
k_{p i}\left(\tilde{q}_{i}, \dot{q}_{i}\right)=k_{p i} \cos ^{2}\left(\tanh \left(\tilde{q}_{i}+\dot{q}_{i}\right)\right) \\
k_{v i}\left(\tilde{q}_{i}, \dot{q}_{i}\right)=k_{v i} \cos ^{2}\left(\tanh \left(\tilde{q}_{i}+\dot{q}_{i}\right)\right) \\
k_{p i}\left(\tilde{q}_{i}, \dot{q}_{i}\right)=k_{p i} \cos ^{2}\left(\frac{\sinh \left(\tilde{q}_{i}+\dot{q}_{i}\right)}{1+\cosh \left(\dot{q}_{i}+\tilde{q}_{i}\right)}\right) \\
k_{v i}\left(\tilde{q}_{i}, \dot{q}_{i}\right)=k_{v i} \cos ^{2}\left(\frac{\sinh \left(\tilde{q}_{i}+\dot{q}_{i}\right)}{1+\cosh \left(\dot{q}_{i}+\tilde{q}_{i}\right)}\right) \\
k_{p i}\left(\tilde{q}_{i}, \dot{q}_{i}\right)=k_{p i} \cos ^{2}\left(\frac{\tilde{q}_{i}}{\sqrt{1+\tilde{q}_{i}^{2}}}\right) \\
k_{v i}\left(\tilde{q}_{i}, \dot{q}_{i}\right)=k_{v i} \cos ^{2}\left(\frac{\tilde{q}_{i}}{\sqrt{1+\tilde{q}_{i}^{2}}}\right)
\end{gathered}
$$

where $k_{p i}\left(\tilde{q}_{i}, \dot{q}_{i}\right)$ and $k_{v i}\left(\tilde{q}_{i}, \dot{q}_{i}\right)$ are inputs of proportional and derivative diagonal matrices. In Fig. 1, can be observed the cases (17), (18) and (19). Note that, even the different proposal of the cases $f_{p}\left(\tilde{q}_{i}, \dot{q}_{i}\right)$ and $f_{p}\left(\tilde{q}_{i}, \dot{q}_{i}\right)$, the proportional and derivative have a very similar behavior (see Fig. 1); for this reason, is possible to propose a gamma of variable gains through definition presented. At the same time, the variable gains curve allows a soft self-tuning where the joint position error and velocity tend to zero.

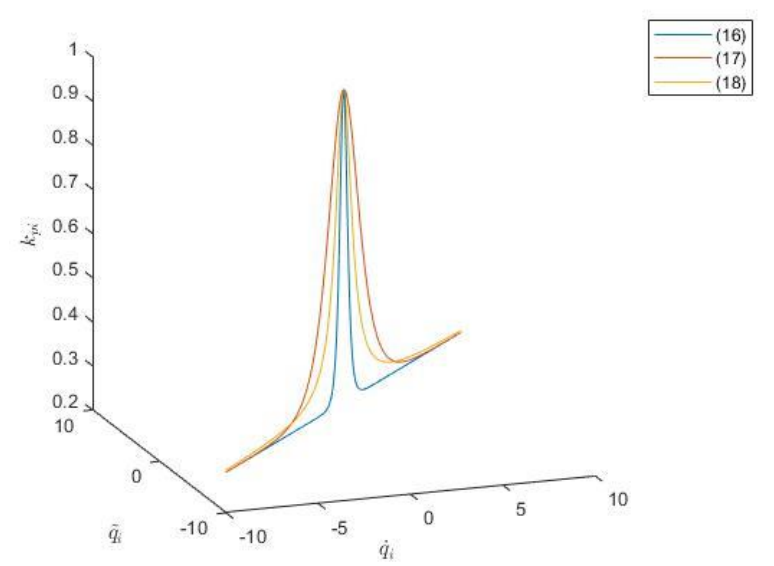

Fig. 1. Gamma of proportional and derivative variable gains.

\section{SATURED CONTROLS WITH VARIABLE GAINS}

The regulation problem in joint space for robot manipulators is presented in (20), which consists in propose a scheme control such that, the applied torque $\boldsymbol{\tau} \in \mathbb{R}^{n}$ to the robot joints $\boldsymbol{q}(t) \in \mathbb{R}^{n}$ tends asymptotically to the desired position vector $\boldsymbol{q}_{\boldsymbol{d}} \in \mathbb{R}^{n}$, regardless the initial conditions $[\widetilde{\boldsymbol{q}}(0), \dot{\boldsymbol{q}}(0)]^{T} \in \mathbb{R}^{n}$ :

$$
\lim _{t \rightarrow \infty}\left[\begin{array}{c}
\widetilde{\boldsymbol{q}}(t) \\
\dot{\boldsymbol{q}}(t)
\end{array}\right] \rightarrow \mathbf{0} \in \mathbb{R}^{n}
$$

for all $t \geq 0$.

The control schemes presented in (21) are a solution to regulation problem (20) and the practical limitation of servomotors for robot manipulator. These control schemes can generate bounded torque by proportional saturation obtained from energy shaping of the artificial potential energy minus a damping injection term saturating the derivative control action with hyperbolic and trigonometric functions. Finally, we add a gravity compensation. This represents a great advantage for Lyapunov stability analysis and practical performance on robot manipulators:

$$
\boldsymbol{\tau}=K_{p}(\widetilde{\boldsymbol{q}}, \dot{\boldsymbol{q}})\left[\begin{array}{c}
\frac{\arctan \left(\alpha \tilde{q}_{1}\right)}{\sqrt{1+\tanh ^{2}\left(\alpha \tilde{q}_{1}\right)}} \\
\frac{\arctan \left(\alpha \tilde{q}_{2}\right)}{\sqrt{1+\tanh ^{2}\left(\alpha \tilde{q}_{2}\right)}} \\
\vdots \\
\frac{\arctan \left(\alpha \tilde{q}_{n}\right)}{\sqrt{1+\tanh ^{2}\left(\alpha \tilde{q}_{n}\right)}}
\end{array}\right]-K_{v}(\widetilde{\boldsymbol{q}}, \dot{\boldsymbol{q}})\left[\begin{array}{c}
\frac{\arctan \left(\alpha \dot{q}_{1}\right)}{\sqrt{1+\tanh ^{2}\left(\alpha \dot{q}_{1}\right)}} \\
\frac{\arctan \left(\alpha \dot{q}_{2}\right)}{\sqrt{1+\tanh ^{2}\left(\alpha \dot{q}_{2}\right)}} \\
\vdots \\
\frac{\arctan \left(\alpha \dot{q}_{n}\right)}{\sqrt{1+\tanh ^{2}\left(\alpha \dot{q}_{n}\right)}}
\end{array}\right]+\boldsymbol{g}(\boldsymbol{q})
$$

being $\alpha$ a positive constant such that, control scheme is modified. This is illustrated in Fig. 2. Can be observed, we have a different control actions when $\alpha$ value is changed. In the proportional term, while $\alpha$ increases, the slope becomes vertical and is approached to quickly drive the position error to zero. 


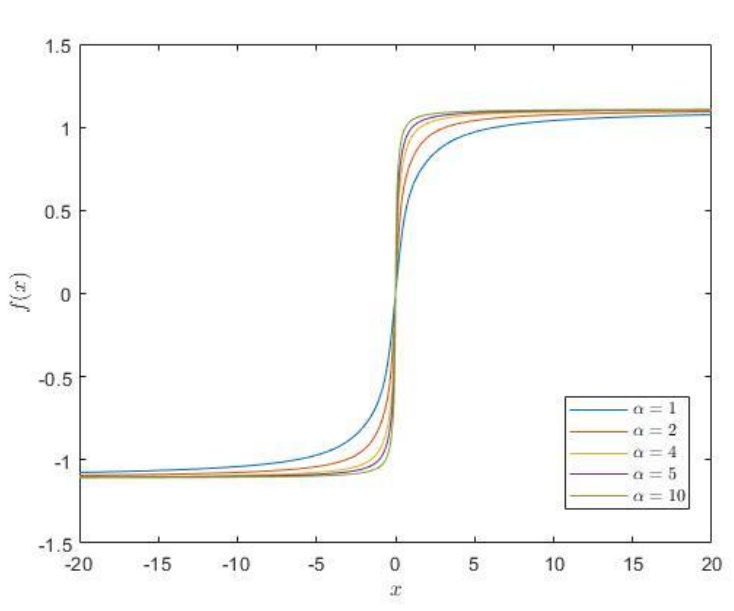

Fig. 2. Saturated scheme controls.

On the other hand, when $\alpha$ is increased, the energy dissipation of the derivative term is obtained; this helps to avoid over oscillations in transitory regime. Also, mathematically is satisfied:

$$
\sqrt{n} \mu_{\alpha} \leq\left\|\mid \begin{array}{c}
\frac{\arctan \left(\alpha x_{1}\right)}{\sqrt{1+\tanh ^{2}\left(\alpha x_{1}\right)}} \\
\frac{\arctan \left(\alpha x_{2}\right)}{\sqrt{1+\tanh ^{2}\left(\alpha x_{2}\right)}} \\
\vdots \\
\frac{\arctan _{\alpha}\left(\alpha x_{n}\right)}{\sqrt{1+\tanh ^{2}\left(\alpha x_{n}\right)}}
\end{array}\right\| \| \begin{gathered}
\sqrt{n} \beta_{\alpha} ; \forall \boldsymbol{x} \in \mathbb{R}^{n} \\
\sqrt{n} \beta_{\alpha}\|\boldsymbol{x}\| ; \forall \boldsymbol{x} \in \mathbb{R}^{n}
\end{gathered}
$$

being $\beta_{\alpha}$ and $\mu_{\alpha}$ the upper and lower bound, respectively, depending on $\alpha, n \in \mathbb{N}$ is a positive integer number. For the proportional and derivative term, we rename as $\beta_{p \alpha}, \mu_{p \alpha}$, and $\beta_{v \alpha}, \mu_{v \alpha}$, respectively.

The close-loop system equation is formed by the robot dynamics (1) and the control law (21). We obtain:

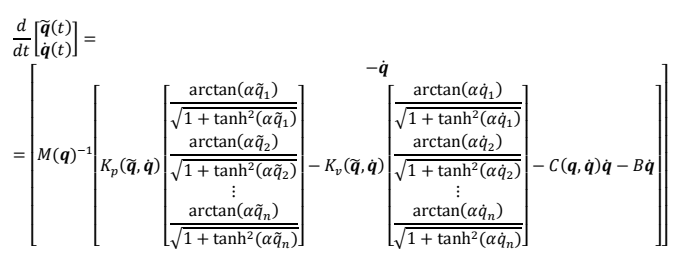

which is an autonomous nonlinear differential equation where the origin $[\widetilde{\boldsymbol{q}}, \dot{\boldsymbol{q}}]^{T}=\mathbf{0} \in \mathbb{R}^{2 n}$ is the unique equilibrium point, under propriety 2.1 and propriety 2.2.

\section{A. Lyapunov stability analysis}

The equilibrium point $[\widetilde{\boldsymbol{q}}, \dot{\boldsymbol{q}}]^{T}=\mathbf{0} \in \mathbb{R}^{2 n}$ of the closeloop system equation (22) is globally asymptotically stable in Lyapunov theory context. In the order to demonstrate said stability, we propose a strict Lyapunov function which is a positive definite and its derivate is negative definite, as follows:

$$
\begin{aligned}
& V(\widetilde{\boldsymbol{q}}, \dot{\boldsymbol{q}})=\frac{1}{2} \dot{\boldsymbol{q}}^{T} M(\boldsymbol{q}) \dot{\boldsymbol{q}}+ \int_{\mathbf{0}}^{\widetilde{q}}\left[\begin{array}{c}
\frac{\arctan \left(\alpha \sigma_{1}\right)}{\sqrt{1+\tanh ^{2}\left(\alpha \sigma_{1}\right)}} \\
\frac{\arctan \left(\alpha \sigma_{2}\right)}{\sqrt{1+\tanh ^{2}\left(\alpha \sigma_{2}\right)}} \\
\vdots \\
\frac{\arctan \left(\alpha \sigma_{n}\right)}{\sqrt{1+\tanh ^{2}\left(\alpha \sigma_{n}\right)}}
\end{array}\right]^{T} K_{p}(\boldsymbol{\sigma}, \dot{\boldsymbol{q}}) d \boldsymbol{\sigma} \\
&-\frac{\epsilon_{0}}{1+\|\widetilde{\boldsymbol{q}}\|} \widetilde{\boldsymbol{q}}^{T} M(\boldsymbol{q}) \dot{\boldsymbol{q}}
\end{aligned}
$$

$\epsilon_{0}$ is a positive constant such that satisfies (25); therefore, the Lyapunov function candidate (24) is a positive definite function.

$$
\frac{\sqrt{k_{\boldsymbol{p l}} \mu_{p \alpha} \lambda_{\min }\{M(\boldsymbol{q})\}}}{\lambda_{\operatorname{Max}}\{M(\boldsymbol{q})\}}[1+\|\widetilde{\boldsymbol{q}}\|]>\epsilon_{0}>0
$$

Proof 2 Note that, the following is satisfied:

$$
\begin{aligned}
\frac{1}{2} \dot{\boldsymbol{q}}^{T} M(\boldsymbol{q}) \dot{\boldsymbol{q}} & =\left|\frac{1}{2} \dot{\boldsymbol{q}}^{T} M(\boldsymbol{q}) \dot{\boldsymbol{q}}\right| \\
& \geq \frac{1}{2} \lambda_{\min }\{M(\boldsymbol{q})\}\|\dot{\boldsymbol{q}}\|\|\dot{\boldsymbol{q}}\|
\end{aligned}
$$

Using property 2.6 and the expression (22), we have:

$$
\begin{gathered}
\int_{0}^{\tilde{q}}\left[\begin{array}{c}
\frac{\arctan \left(\alpha \sigma_{1}\right)}{\sqrt{1+\tanh ^{2}\left(\alpha \sigma_{1}\right)}} \\
\frac{\arctan \left(\alpha \sigma_{2}\right)}{\sqrt{1+\tanh ^{2}\left(\alpha \sigma_{2}\right)}} \\
\vdots \\
\frac{\arctan \left(\alpha \sigma_{n}\right)}{\sqrt{1+\tanh ^{2}\left(\alpha \sigma_{n}\right)}}
\end{array}\right]^{T} \quad K_{p}(\boldsymbol{\sigma}, \dot{\boldsymbol{q}}) d \boldsymbol{\sigma} \geq \frac{1}{2} k_{p l}\left\|\left[\begin{array}{c}
\frac{\arctan \left(\alpha \tilde{q}_{1}\right)}{\sqrt{1+\tanh ^{2}\left(\alpha \tilde{q}_{1}\right)}} \\
\frac{\arctan \left(\alpha \tilde{q}_{2}\right)}{\sqrt{1+\tanh ^{2}\left(\alpha \tilde{q}_{2}\right)}} \\
\vdots \\
\frac{\arctan \left(\alpha \tilde{q}_{n}\right)}{\sqrt{1+\tanh ^{2}\left(\alpha \tilde{q}_{n}\right)}}
\end{array}\right]\right\|\|\widetilde{\boldsymbol{q}}\| \\
\\
\geq \frac{1}{2} k_{\boldsymbol{p l}} \sqrt{n} \mu_{p \alpha}\|\widetilde{\boldsymbol{q}}\|^{2}
\end{gathered}
$$

Also:

$$
-\frac{\epsilon_{0}}{1+\|\widetilde{\boldsymbol{q}}\|} \widetilde{\boldsymbol{q}}^{T} M(\boldsymbol{q}) \dot{\boldsymbol{q}} \geq \frac{\epsilon_{0}}{1+\|\tilde{\boldsymbol{q}}\|} \lambda_{M a x}\{M(\boldsymbol{q})\}\|\widetilde{\boldsymbol{q}}\|\|\dot{\boldsymbol{q}}\|
$$

Under before equations, Lyapunov function is bounded inferiorly as expression (29):

$$
\begin{aligned}
V(\widetilde{\boldsymbol{q}}, \dot{\boldsymbol{q}}) \geq \frac{1}{2} k_{\boldsymbol{p l}} \sqrt{n} \mu_{p \alpha}\|\widetilde{\boldsymbol{q}}\|^{2}+\frac{1}{2} \lambda_{\min }\{M(\boldsymbol{q})\}\|\dot{\boldsymbol{q}}\|^{2} \\
-\frac{\epsilon_{0}}{1+\|\widetilde{\boldsymbol{q}}\|} \lambda_{M a x}\{M(\boldsymbol{q})\}\|\widetilde{\boldsymbol{q}}\|\|\dot{\boldsymbol{q}}\|
\end{aligned}
$$

and rewritten in the quadratic form:

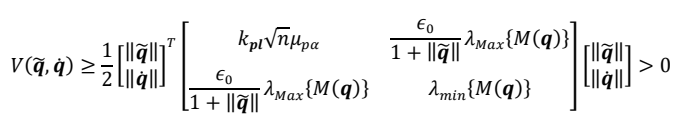

for that (30) is a positive definite function if $\epsilon_{0}$ exists in the interval (31):

$$
\frac{\sqrt{k_{p l} \sqrt{n} \mu_{p \alpha} \lambda_{\min }\{M(\boldsymbol{q})\}}}{\lambda_{\operatorname{Max}}\{M(\boldsymbol{q})\}}[1+\|\widetilde{\boldsymbol{q}}\|]>\epsilon_{0}>0
$$

We continue to obtain the time derivative of Lyapunov function (24) along the trajectories of the close-loop equation (23). By the substitution of joint acceleration $\ddot{\boldsymbol{q}} \in \mathbb{R}^{n}$, using propriety 2.3 , propriety 2.4 , the Leibniz rule for the 
derivation of integrals and algebraical cancellation of terms, we have:

$$
\begin{aligned}
& \dot{V}(\widetilde{\boldsymbol{q}}, \dot{\boldsymbol{q}})=\dot{\boldsymbol{q}}^{T} M(\boldsymbol{q}) \ddot{\boldsymbol{q}}+\frac{1}{2} \dot{\boldsymbol{q}}^{T} \dot{M}(\boldsymbol{q}) \dot{\boldsymbol{q}}+\dot{\boldsymbol{q}}^{T} K_{p}(\widetilde{\boldsymbol{q}}, \dot{\boldsymbol{q}})\left[\begin{array}{c}
\frac{\arctan \left(\alpha \tilde{q}_{1}\right)}{\sqrt{1+\tanh ^{2}\left(\alpha \tilde{q}_{1}\right)}} \\
\frac{\arctan \left(\alpha \tilde{q}_{2}\right)}{\sqrt{1+\tanh ^{2}\left(\alpha \tilde{q}_{2}\right)}} \\
\vdots \\
\frac{\arctan \left(\alpha \tilde{q}_{n}\right)}{\sqrt{1+\tanh ^{2}\left(\alpha \tilde{q}_{n}\right)}}
\end{array}\right] \\
& -\frac{\epsilon_{0}}{1+\|\widetilde{\boldsymbol{q}}\|} \widetilde{\boldsymbol{q}}^{T} M(\boldsymbol{q}) \ddot{\boldsymbol{q}}-\frac{\epsilon_{0}}{1+\|\widetilde{\boldsymbol{q}}\|} \widetilde{\boldsymbol{q}}^{T} \dot{M}(\boldsymbol{q}) \dot{\boldsymbol{q}} \\
& -\frac{\epsilon_{0}}{1+\|\widetilde{\boldsymbol{q}}\|} \dot{\tilde{\boldsymbol{q}}}^{T} M(\boldsymbol{q}) \dot{\boldsymbol{q}}-\frac{\epsilon_{0} \widetilde{\boldsymbol{q}}^{T} \dot{\boldsymbol{q}}}{\|\widetilde{\boldsymbol{q}}\|[1+\|\widetilde{\boldsymbol{q}}\|]^{2}} \widetilde{\boldsymbol{q}}^{T} M(\boldsymbol{q}) \dot{\boldsymbol{q}} \\
& \dot{V}(\widetilde{\boldsymbol{q}}, \dot{\boldsymbol{q}})=-\dot{\boldsymbol{q}}^{T} B \dot{\boldsymbol{q}}-\dot{\boldsymbol{q}}^{T} K_{v}(\widetilde{\boldsymbol{q}}, \dot{\boldsymbol{q}})\left[\begin{array}{c}
\frac{\arctan \left(\alpha \dot{q}_{1}\right)}{\sqrt{1+\tanh \left(\alpha \dot{q}_{1}\right)}} \\
\frac{\arctan \left(\alpha \dot{q}_{2}\right)}{\sqrt{1+\tanh ^{2}\left(\alpha \dot{q}_{2}\right)}} \\
\vdots \\
\frac{\arctan \left(\alpha \dot{q}_{n}\right)}{\sqrt{1+\tanh ^{2}\left(\alpha \dot{q}_{n}\right)}}
\end{array}\right]-\frac{\epsilon_{0}}{1+\|\widetilde{\boldsymbol{q}}\|} \widetilde{\boldsymbol{q}}^{T} M(\boldsymbol{q}) \ddot{\boldsymbol{q}} \\
& -\frac{\epsilon_{0}}{1+\|\widetilde{\boldsymbol{q}}\|^{\mid}} \widetilde{\boldsymbol{q}}^{T}\left[C(\boldsymbol{q}, \boldsymbol{q})+C(\boldsymbol{q}, \dot{\boldsymbol{q}})^{T}\right] \dot{\boldsymbol{q}} \\
& +\frac{\epsilon_{0}}{1+\|\widetilde{\boldsymbol{q}}\|} \dot{\boldsymbol{q}}^{T} M(\boldsymbol{q}) \dot{\boldsymbol{q}}-\frac{\epsilon_{0} \widetilde{\boldsymbol{q}}^{T} \dot{\boldsymbol{q}}}{\|\widetilde{\boldsymbol{q}}\|[1+\|\widetilde{\boldsymbol{q}}\|]^{2}} \widetilde{\boldsymbol{q}}^{T} M(\boldsymbol{q}) \dot{\boldsymbol{q}} \\
& \dot{V}(\widetilde{\boldsymbol{q}}, \dot{\boldsymbol{q}})=-\dot{\boldsymbol{q}}^{T} B \dot{\boldsymbol{q}}-\dot{\boldsymbol{q}}^{T} K_{v}(\widetilde{\boldsymbol{q}}, \dot{\boldsymbol{q}})\left[\begin{array}{c}
\frac{\arctan \left(\alpha \dot{q}_{1}\right)}{\sqrt{1+\tanh \left(\alpha \dot{\alpha} \dot{1}_{1}\right)}} \\
\frac{\arctan \left(\alpha \dot{q}_{2}\right)}{\sqrt{1+\tanh ^{2}\left(\alpha \dot{q}_{2}\right)}} \\
\vdots \\
\frac{\arctan \left(\alpha \dot{q}_{n}\right)}{\sqrt{1+\tanh ^{2}\left(\alpha \dot{q}_{n}\right)}}
\end{array}\right]+\frac{\epsilon_{0}}{1+\|\widetilde{\boldsymbol{q}}\|} \widetilde{\boldsymbol{q}}^{T} B \dot{\boldsymbol{q}} \\
& +\frac{\epsilon_{0}}{1+\|\widetilde{\boldsymbol{q}}\|} \widetilde{\boldsymbol{q}}^{T} K_{v}(\widetilde{\boldsymbol{q}}, \boldsymbol{\boldsymbol { q }})\left[\begin{array}{c}
\frac{\arctan \left(\alpha \dot{q}_{1}\right)}{\sqrt{1+\tanh ^{2}\left(\alpha \dot{q}_{1}\right)}} \\
\frac{\arctan \left(\alpha \dot{q}_{2}\right)}{\sqrt{1+\tanh ^{2}\left(\alpha \dot{q}_{2}\right)}} \\
\vdots \\
\frac{\arctan \left(\alpha \dot{q}_{n}\right)}{\sqrt{1+\tanh ^{2}\left(\alpha \dot{q}_{n}\right)}}
\end{array}\right] \\
& -\frac{\epsilon_{0}}{1+\|\widetilde{\boldsymbol{q}}\|} \widetilde{\boldsymbol{q}}^{T} K_{p}(\widetilde{\boldsymbol{q}}, \dot{\boldsymbol{q}})\left[\begin{array}{c}
\frac{\arctan \left(\alpha \widetilde{q}_{1}\right)}{\sqrt{1+\tanh ^{2}\left(\alpha \widetilde{q}_{1}\right)}} \\
\frac{\arctan \left(\alpha \widetilde{q}_{2}\right)}{\sqrt{1+\tanh ^{2}\left(\alpha \widetilde{q}_{2}\right)}} \\
\vdots \\
\frac{\arctan \left(\alpha \widetilde{q}_{n}\right)}{\sqrt{1+\tanh ^{2}\left(\alpha \widetilde{q}_{n}\right)}}
\end{array}\right] \\
& -\frac{\epsilon_{0}}{1+\|\widetilde{\boldsymbol{q}}\|} \widetilde{\boldsymbol{q}}^{T}\left[C(\boldsymbol{q}, \dot{\boldsymbol{q}})^{T}\right] \dot{\boldsymbol{q}}+\frac{\epsilon_{0}}{1+\|\widetilde{\boldsymbol{q}}\|} \dot{\boldsymbol{q}}^{T} M(\boldsymbol{q}) \dot{\boldsymbol{q}} \\
& -\frac{\epsilon_{0} \widetilde{\boldsymbol{q}}^{T} \dot{\boldsymbol{q}}}{\|\widetilde{\boldsymbol{q}}\|[1+\|\widetilde{\boldsymbol{q}}\|]^{2}} \widetilde{\boldsymbol{q}}^{T} M(\boldsymbol{q}) \dot{\boldsymbol{q}}
\end{aligned}
$$

Take propriety 2.5 and (21), the equation (34) is bounded superiorly as following:

$$
\begin{aligned}
\dot{V}(\widetilde{\boldsymbol{q}}, \dot{\boldsymbol{q}}) \leq-\lambda_{\min }\{B\}\|\dot{\boldsymbol{q}}\|^{2}- & \lambda_{\min }\left\{K_{v}(\widetilde{\boldsymbol{q}}, \dot{\boldsymbol{q}})\right\} \sqrt{n} \beta_{v \alpha}\|\dot{\boldsymbol{q}}\|^{2} \\
& +\frac{\epsilon_{0}}{1+\|\widetilde{\boldsymbol{q}}\|} \lambda_{M a x}\{B\}\|\widetilde{\boldsymbol{q}}\|\|\dot{\boldsymbol{q}}\| \\
& +\frac{\epsilon_{0}}{1+\|\widetilde{\boldsymbol{q}}\|} \lambda_{\text {Max }}\left\{K_{v}(\widetilde{\boldsymbol{q}}, \dot{\boldsymbol{q}})\right\} \sqrt{n} \beta_{v \alpha}\|\widetilde{\boldsymbol{q}}\|\|\dot{\boldsymbol{q}}\| \\
& -\frac{\epsilon_{0}}{1+\|\widetilde{\boldsymbol{q}}\|} \lambda_{\min }\left\{K_{p}(\widetilde{\boldsymbol{q}}, \dot{\boldsymbol{q}})\right\} \sqrt{n} \beta_{p \alpha}\|\dot{\boldsymbol{q}}\|^{2}-\epsilon_{0} k_{c}\|\dot{\boldsymbol{q}}\|^{2} \\
& +\frac{\epsilon_{0}}{1+\|\widetilde{\boldsymbol{q}}\|} \lambda_{\text {Max }}\{M(\boldsymbol{q})\}\|\dot{\boldsymbol{q}}\|^{2} \\
& -\frac{\epsilon_{0}}{1+\|\widetilde{\boldsymbol{q}}\|} \lambda_{\min }\{M(\boldsymbol{q})\}\|\widetilde{\boldsymbol{q}}\|\|\dot{\boldsymbol{q}}\|
\end{aligned}
$$

then:

$$
\dot{V}(\widetilde{\boldsymbol{q}}, \dot{\boldsymbol{q}}) \leq-\left[\begin{array}{l}
\|\widetilde{\boldsymbol{q}}\| \\
\|\dot{\boldsymbol{q}}\|
\end{array}\right]^{T} \mathrm{Z}\left[\begin{array}{l}
\|\widetilde{\boldsymbol{q}}\| \\
\|\dot{\boldsymbol{q}}\|
\end{array}\right]<0
$$

where $\mathrm{Z} \in \mathbb{R}^{2 \times 2}$ with the elements:

- $\quad z_{11}=\frac{\epsilon_{0}}{1+\|\widetilde{\boldsymbol{q}}\|} \lambda_{\min }\left\{K_{p}(\widetilde{\boldsymbol{q}}, \dot{\boldsymbol{q}})\right\} \sqrt{n} \beta_{p \alpha}$

- $z_{12}=\frac{1}{2} \frac{\epsilon_{0}}{1+\|\widetilde{\boldsymbol{q}}\|}\left[\lambda_{\min }\{M(\boldsymbol{q})\}-\lambda_{\text {Max }}\left\{K_{v}(\widetilde{\boldsymbol{q}}, \dot{\boldsymbol{q}})\right\} \sqrt{n}-\lambda_{\text {Max }}\{B\}\right]$
- $\quad z_{21}=z_{12}$

- $\quad z_{21}=\lambda_{\min }\{B\}+\lambda_{\min }\left\{K_{v}(\widetilde{\boldsymbol{q}}, \dot{\boldsymbol{q}})\right\} \sqrt{n} \beta_{v \alpha}+$ $\frac{\epsilon_{0}}{1+\|\widetilde{\boldsymbol{q}}\|} \lambda_{\min }\left\{K_{p}(\widetilde{\boldsymbol{q}}, \dot{\boldsymbol{q}})\right\} \sqrt{n} \beta_{p \alpha}+\epsilon_{0} k_{c}\|\dot{\boldsymbol{q}}\|^{2}-\frac{\epsilon_{0}}{1+\|\widetilde{\boldsymbol{q}}\|} \lambda_{M a x}\{M(\boldsymbol{q})\}$

To ensure that (36) can be a positive definite function, the determinant of the matrix $\mathrm{Z}$ must be positive: $\operatorname{det}[\mathrm{Z}]>0$; this condition is satisfied for any $\epsilon_{0}$ on the next interval:

$$
\begin{gathered}
\frac{\lambda_{\min }\left\{K_{p}(\widetilde{\boldsymbol{q}}, \dot{\boldsymbol{q}})\right\} \sqrt{n} \beta_{p \alpha}\left[N_{1}\right]}{\lambda_{\min }\left\{K_{p}(\widetilde{\boldsymbol{q}}, \dot{\boldsymbol{q}})\right\} \sqrt{n} \beta_{p \alpha}\left[D_{1}\right]+\frac{1}{4}\left[D_{2}\right]^{2}}[1+\|\widetilde{\boldsymbol{q}}\|]>\epsilon_{0}>0 \\
\text { - } \quad N_{1}=\frac{\epsilon_{0}}{1+\|\widetilde{\boldsymbol{q}}\|} \lambda_{\min }\left\{K_{p}(\widetilde{\boldsymbol{q}}, \dot{\boldsymbol{q}})\right\} \sqrt{n} \beta_{p \alpha} \\
\text { - } \quad D_{1}=\lambda_{\min }\left\{K_{p}(\widetilde{\boldsymbol{q}}, \dot{\boldsymbol{q}})\right\} \sqrt{n} \beta_{p \alpha}-\lambda_{M a x}\{M(\boldsymbol{q})\} \\
\text { - } \quad D_{2}=\lambda_{\min }\{M(\boldsymbol{q})\}-\lambda_{M a x}\left\{K_{v}(\widetilde{\boldsymbol{q}}, \dot{\boldsymbol{q}})\right\} \sqrt{n} \beta_{v \alpha}-\lambda_{\operatorname{Max}}\{B\}
\end{gathered}
$$

Then $\dot{V}(\widetilde{\boldsymbol{q}}, \dot{\boldsymbol{q}})$ is a negative definite function and $V(\widetilde{\boldsymbol{q}}, \dot{\boldsymbol{q}})$ a positive definite function, if $\epsilon_{0}$ satisfies (25) and (37), simultaneously. Furthermore, $\epsilon_{0}$ numeric value is not necessary, just demonstrate its existence for analysis stability. Therefore, according to Lyapunov direct method, the origin of the close-loop system equation $[\widetilde{\boldsymbol{q}}, \dot{\boldsymbol{q}}]^{T}=\mathbf{0} \in \mathbb{R}^{2 n}$ is globally asymptotically stable: then, both states variables $\widetilde{\boldsymbol{q}}(t)$ and $\dot{\boldsymbol{q}}(t)$ asymptotically converge to zero, when $t \rightarrow \infty$.

\section{DESING OF SELF-TUNING LAW}

We have designed a self-tuning law for variable gains and saturated control schemes of robot manipulators. The general idea is to propose a mathematically rule to determine the coefficients from the gamma of variable gain cases. In this sense, it is simple to be implement by any user. The selftuning law is presented in (38) for the cases of variable gains:

$$
\begin{gathered}
k_{p i}\left(\tilde{q}_{i}, \dot{q}_{i}\right)=k_{p i} \cos ^{2}\left(\tanh \left(\tilde{q}_{i}+\dot{q}_{i}\right)\right) \\
k_{v i}\left(\tilde{q}_{i}, \dot{q}_{i}\right)=k_{v i} \cos ^{2}\left(\tanh \left(\tilde{q}_{i}+\dot{q}_{i}\right)\right) \\
k_{p i}\left(\tilde{q}_{i}, \dot{q}_{i}\right)=k_{p i} \cos ^{2}\left(\frac{\sinh \left(\tilde{q}_{i}\right)}{1+\cosh \left(\dot{q}_{i}\right)}\right) \\
k_{v i}\left(\tilde{q}_{i}, \dot{q}_{i}\right)=k_{v i} \cos ^{2}\left(\frac{\sinh \left(\tilde{q}_{i}\right)}{1+\cosh \left(\dot{q}_{i}\right)}\right) \\
k_{p i}\left(\tilde{q}_{i}, \dot{q}_{i}\right)=k_{p i} \cos ^{2}\left(\frac{\tilde{q}_{i}}{\sqrt{1+\dot{q}_{i}}}\right) \\
k_{v i}\left(\tilde{q}_{i}, \dot{q}_{i}\right)=k_{v i} \cos ^{2}\left(\frac{\tilde{q}_{i}}{\sqrt{1+\dot{q}_{i}}}\right) \\
k_{p i}=2 \delta_{i} \sqrt{\tau_{i}^{M a x}} \\
k_{v i}=\frac{1}{3} \sqrt{\left(k_{p i}+1\right)^{2}}
\end{gathered}
$$

where $\tau_{i}^{\text {Max }}$ is maximum torque applied by the servomotor corresponding to $i^{\text {th }}$ joint of robot manipulator. The constant $\delta_{i}$ lives in the interval $5>\delta_{i}>0$ which is proposed by the user. This constant has as main objective, gives an initial value to proportional variable gains. Thanks to the characteristic curve of variable gains, we ensure numerical changes of control gains until self-tuning is achieved, when $i^{\text {th }}$ joint position error and joint velocity tend to zero. The globally asymptotic stability is demonstrated then, the selftuning is insured. This law allows drive the position error to 
zero and inject damping energy for attenuate oscillations in transitory regime with smoothly permanent regime.

\section{SIMULATION RESULTS}

To support the theoretical results, the performance and effectiveness of the controllers propose, simulation results on two degrees direct-drive-robot (see Fig. 3) are presented in this section. The objective is to verify the functionality of the control schemes, gamma of variable gains with comparative analysis of performance against PD controllers, where the control gains is realized manually. The virtual experiment consists of drive the joint positions to desired joint positions $\left[45^{\circ}, 90^{\circ}\right]^{T}$ since home positions $\left[0^{\circ}, 0^{\circ}\right]^{T}$. Considering $\alpha=$ $1, \alpha=3$ and $\alpha=5$ with variable gains case (17) the robot regulation is implemented. Later, evaluating the cases of variable gains (18) and (19) with $\alpha=5$. The maximum torques applied by each servomotor of the robot manipulator: $\tau_{1}^{M a z}=150 \mathrm{Nm}$ and, $\tau_{1}^{M a z}=15 \mathrm{Nm}$; for shoulder (first joint) and elbow (second joint), respectively. For last, the dynamic parameters of robot manipulator are reported in [30] which are obtained by parametric identification strategics. In this sense, the simulation results can be very similar with experimental results on a direct-drive-robot manipulator. In Table 1 is shown the numeric parameters necessary for selftuning law. Tus, $\delta_{i}$ value is proposed by designer which must be in the interval $5>\delta_{i}>0$.

TABLE I. SELF-TUNING LAW PARAMETERS

\begin{tabular}{ccc}
\hline & Numeric values & \\
\hline Joint & Maximum torque & $\delta_{i}$ \\
\hline Shoulder & $150 \mathrm{Nm}$ & 4.1 \\
\hline Elbow & $15 \mathrm{Nm}$ & 1.5 \\
\hline
\end{tabular}

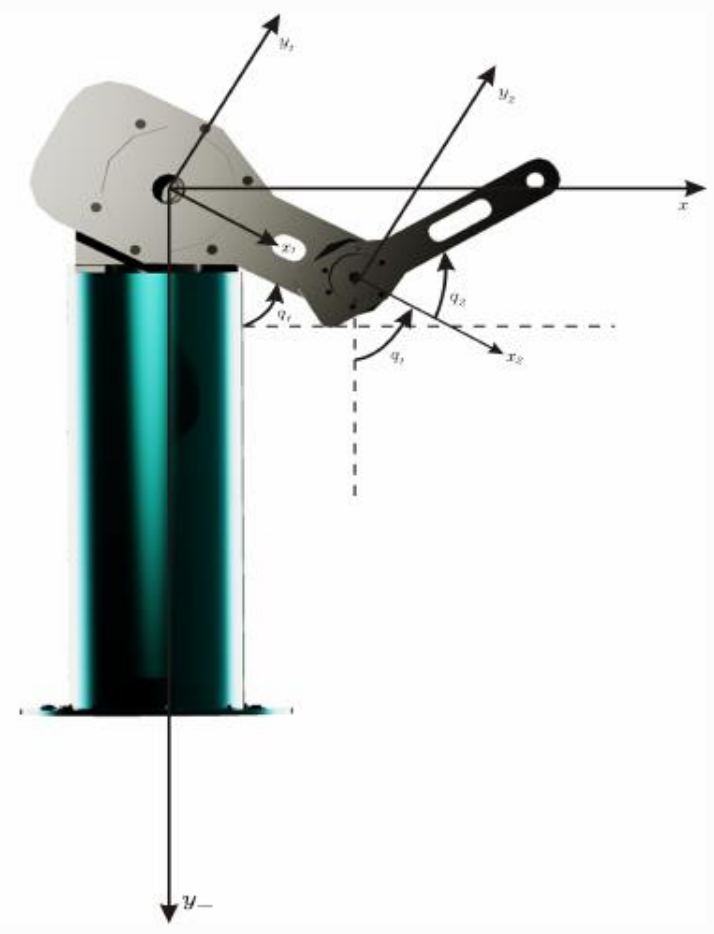

Fig. 3. Two degrees direct-drive robot manipulator.

\section{A. Satured controllers with variable gains}

Fig. 4 contains the position errors of robot manipulator when is regulated by saturated controller with variables gains (18). Note that, each position converges asymptotically to desired position without over-oscillations in transitory regime and quick arrival to permanent regime, less than 3 seconds. After 7.01 seconds, a position error in the order $10^{-7}$ is obtained. This response is attributed to the derivative term through joint velocity and the derivative variable gain. In practical terms, both state variables of the robot manipulator converge to equilibrium point. The self-tuning of variable gains is achieved by the asymptotic convergence to equilibrium point and the correct choice of $\delta_{i}$ numerical value. On the other hand, the applied torques do not exceed the actuators prescribed limits, keeping out of saturation zone, and applying constant energy to robot manipulator and holding on the desired positions, such as is illustrated in Fig. 5 .

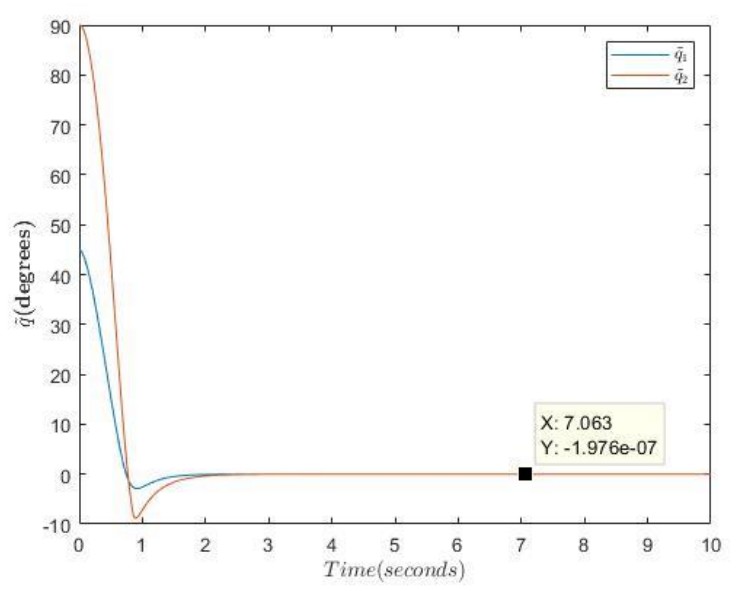

Fig. 4. Position errors of the saturated controller with $\alpha=1$.

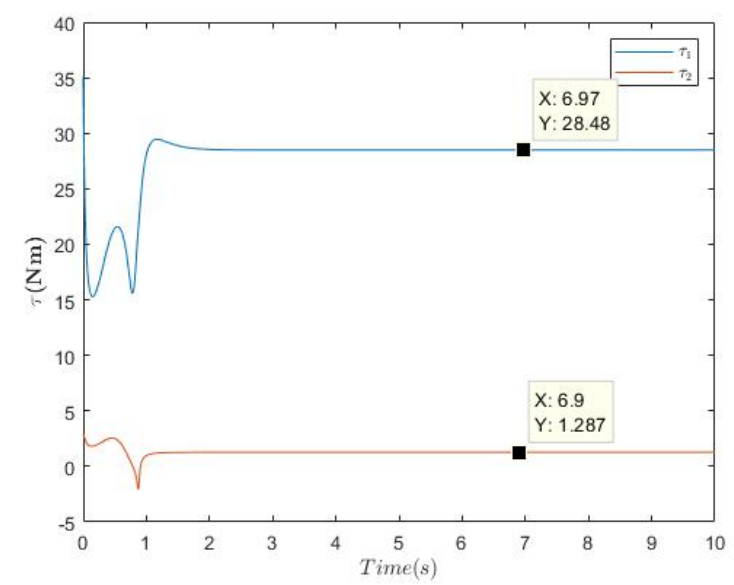

Fig. 5. Applied torques of the saturated controller with $\alpha=1$

Next, $\alpha=3$. In Fig. 6, can be observed the position errors. When $\alpha$ is incremented, the arrival to permanent regime is faster than previous case. In 2 seconds, the joint positions are in the desired position but, have a maximum peak in the transitory regime. Furthermore, the error to permanent regime remains in the order of $10^{-7}$, enough to say that the positions of the robot manipulator are in the 
desired positions. At the same time, all robot states asymptotically converge to equilibrium point.

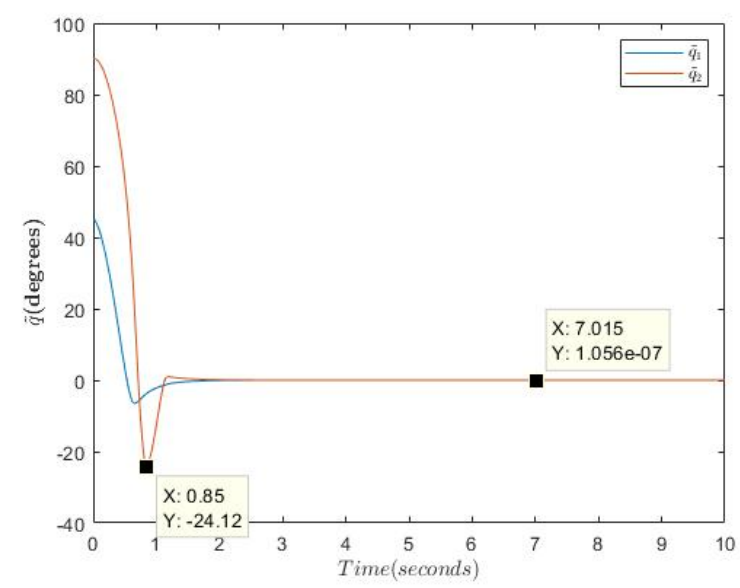

Fig. 6. Position errors of the saturated controller with $\alpha=3$.

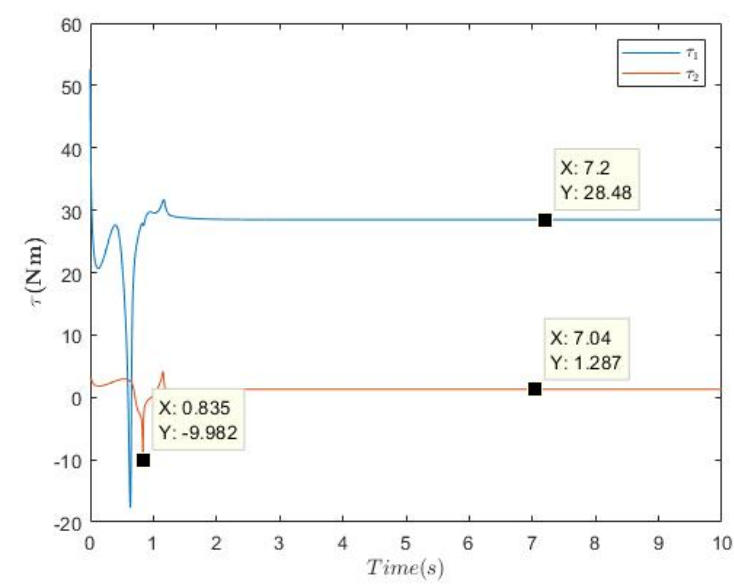

Fig. 7. Applied torques of the saturated controller with $\alpha=3$.

The applied torques is illustrated in Fig. 7. Note that, the torques are the linear zone. Th self-tune of variable gains allows that two types energy can be supplied: energy for drive to desired positions and dissipative energy which acts as artificial mechanical break.

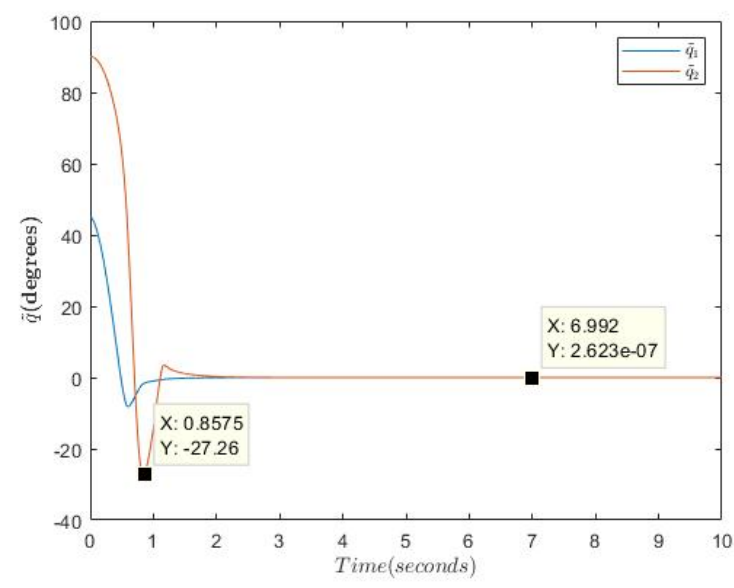

Fig. 8. Position errors of the saturated controller with $\alpha=5$.
To finalize the saturated controls analysis, we consider $\alpha=5$. Observe in Fig. 8 that, the maximum peak increases 7 degrees; however, the position error characteristic curves, is similar. The asymptotic convergence is insured, and variable gains self-tuning is satisfied, even incrementing the $\alpha$ numeric values. The applied torques is under physical limits, previously described (see Fig. 9). Is important to mention, even incrementing $\alpha$ to higher numerical values, the selftuning law provides to the scheme controls for that never exceed any servomotor limits; if, and only if $\delta_{i}$ lives in the proposed interval. In this sense, the selection of this constant is easier than other tuning rule of variable gains for robot manipulators.

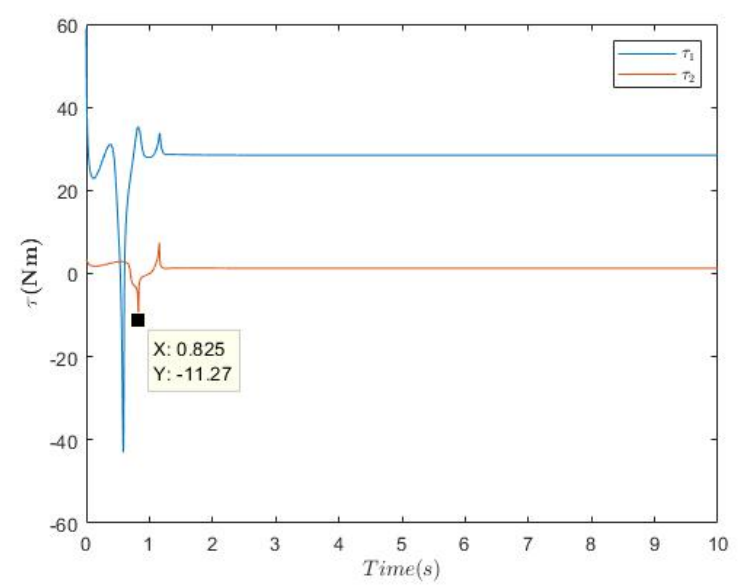

Fig. 9. Applied torques of the saturated controller with $\alpha=5$.

In order to study the variable-gains-gamma functionality, the variable gains case is interchanged by mathematical expression (18) and considering the saturated controller with $\alpha=5$. The change of variable gains case allows that position errors converges to permanent regimen in 2 seconds. In the transitory regime, the maximum peak is increased with respect to previous cases; however, the smooth request, with respect to previous cases, is present in the robot manipulator's joint positions. The self-tuning of variable gains case is insured under design law (38). All before mentioned, is illustrated in Fig. 10.

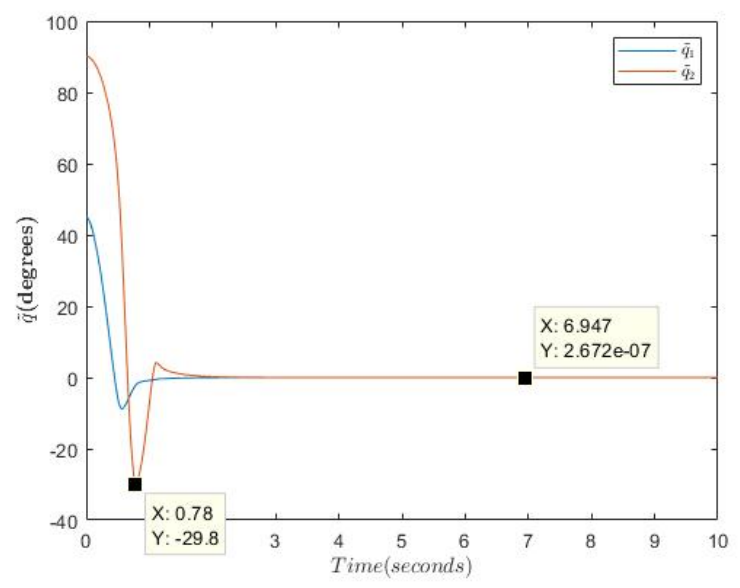

Fig. 10. Position errors of controller with variable gains (17). 
Fig. 11 contains the applied torques by saturated control. We can observe the energy supplied by servomotors of robot manipulator. The prescribed limits do not surpass by the control scheme with variable gains. Out saturation zone, the manipulator does not obtain nonlinearities not considered by robot dynamics. This concept is called unmodeled mechanics. The variable gains are self-tuned such as, the robot manipulator stays in the equilibrium point when $t \rightarrow \infty$.

Finally, the last case of variable gains is evaluated. The mathematical structure of case (19) does not have the hyperbolic functions, with respect to other cases. The main objective is to show that only a bounded function is needed to propose a case of variable gains.

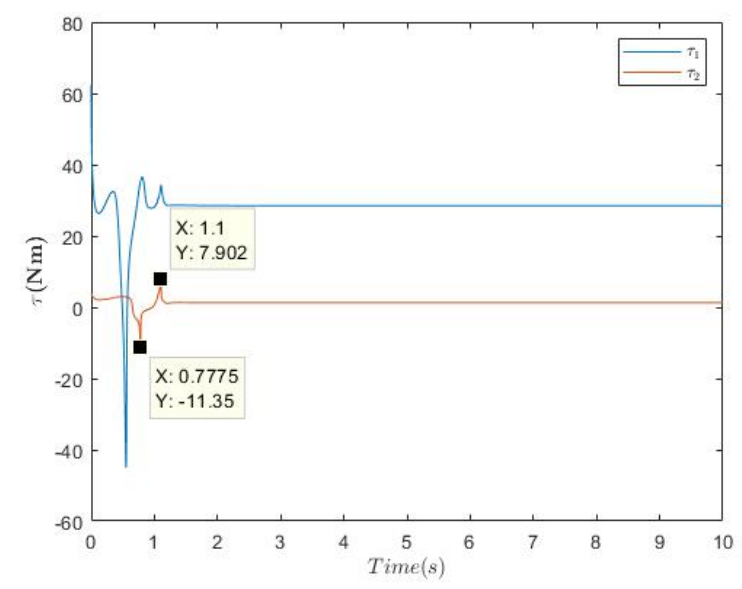

Fig. 11. Applied torques of the controller with variable gains (17).

The position errors are presented in Fig. 12. The transitory regime has not over oscillations due the derivative term and derivative variable gain through joint velocity and position error of robot manipulator. The self-tuning to these variable gains case is satisfied by asymptotic convergence to equilibrium point and design law. Fig. 13 shows the energy applied by controller, where the physical limits are not exceeded. Consequently, the servomotors work in lineal zone.

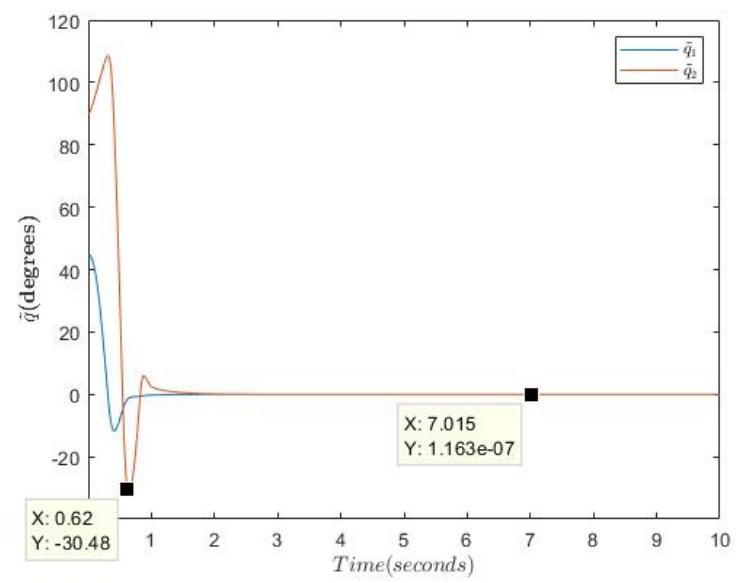

Fig. 12. Position errors of the controller with variable gains (18).

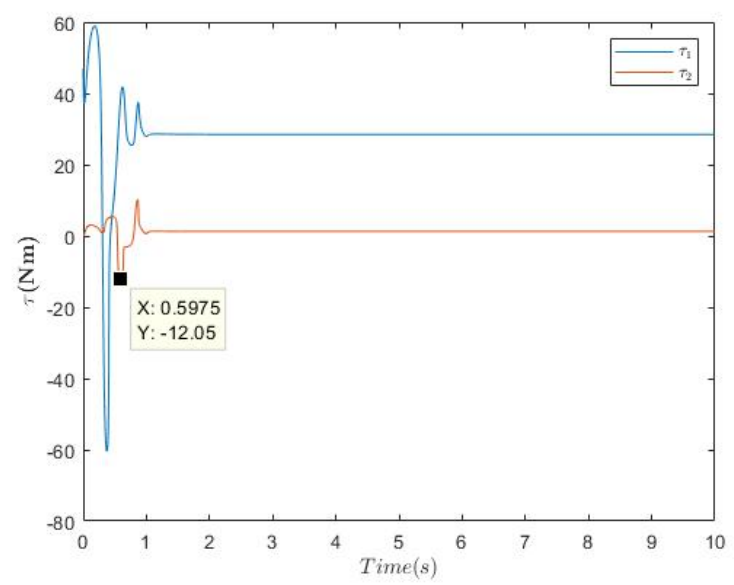

Fig. 13. Applied torques of the controller with variable gains (18).

\section{B. PD control with gravity compensation}

To compare the performance of control schemes and variable gain cases, PD control plus gravity compensation is shown in expression (38) and implemented on the robot manipulator.

$$
\boldsymbol{\tau}=K_{p} \widetilde{\boldsymbol{q}}-K_{v} \dot{\boldsymbol{q}}+\boldsymbol{g}(\boldsymbol{q})
$$

being $K_{p} \in \mathbb{R}^{n \times n}$ and $K_{p} \in \mathbb{R}^{n \times n}$ are diagonal positive define matrix, whose entries are positives. Manually, the tuning is realized. In Fig. 14 and Fig. 15 are shown the position errors and applied torques, respectively. Global regulation of $\mathrm{PD}$ is demonstrated in various papers; therefore, an exhaustive description is not needed. Under Fig. 14 and Fig. 15, the robot manipulator converges to equilibrium point and physical limits is not exceeded.

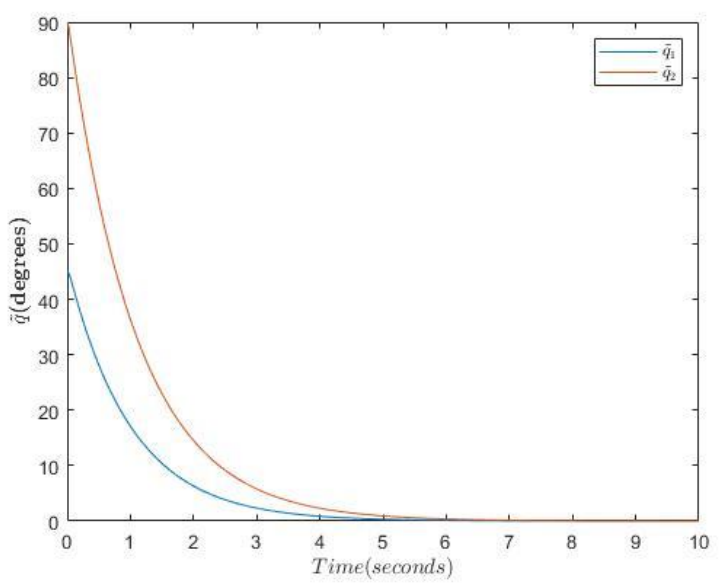

Fig. 14. Position errors of the PD controller. 


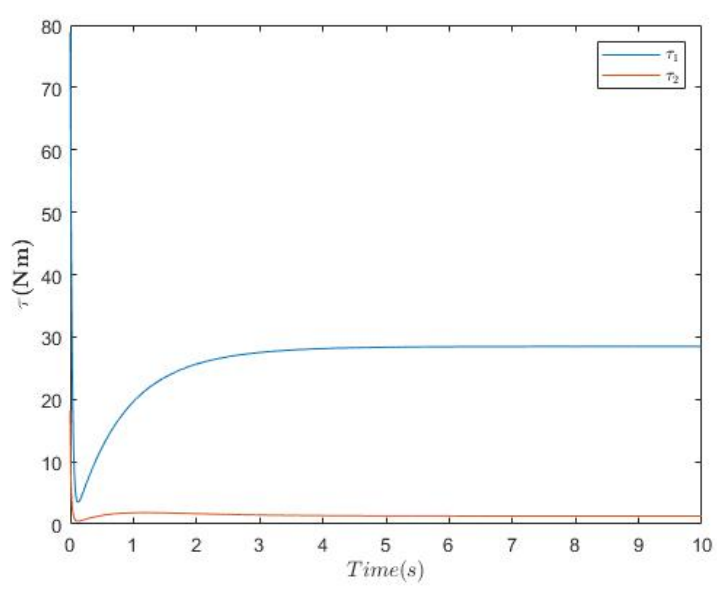

Fig. 15. Applied torques of the PD controller.

\section{Performance evaluation}

The $\mathcal{L}_{2}$ norm is a widely used tool by the scientific community in robotics to measure the control performance. This norm measure performance control through the rootmean-square of the vector of position error. It is given by:

$$
\mathcal{L}_{2}=\sqrt{\frac{1}{T} \int_{0}^{T} \frac{1}{T}\|\widetilde{\boldsymbol{q}}(\sigma)\| d \sigma}
$$

where $T \in \mathbb{R}_{+}$represents the simulation time, in this case 10 seconds. The smaller norm means smaller position error and it is the best evaluated scheme performance.

The controller's evaluation is shown in Fig. 16. The control schemes were renamed to show more clearly the performance indexes of all controllers evaluated (see Table 2 ). When the $\mathcal{L}_{2}$ norm is implemented, the PD control has a $\mathcal{L}_{2}=0.42074$ degrees, Sat 1 with $\mathcal{L}_{2}=0.33035$ degrees, Sat 2 a $\mathcal{L}_{2}=0.35189$ degrees, Sat $3 \mathcal{L}_{2}=0.36374$ degrees, Sat 4 with $\mathcal{L}_{2}=0.3521$ degrees and Sat 5 a $\mathcal{L}_{2}=0.38867$ degrees. Consequently, the performance indexes are organized from highest to lowest. In this sense, the performance quality is evidently observed.

TABLE II. CONTROLLERS EVALUATED

\begin{tabular}{lcc}
\hline & $\begin{array}{c}\text { Control with } \\
\text { variable gains }\end{array}$ & \\
\hline Scheme control & Variable gains & $\alpha$ \\
\hline Saturated (Sat 1) & $(17)$ & 1 \\
\hline Saturated (Sat 2) & $(17)$ & 3 \\
\hline Saturated (Sat 3) & $(17)$ & 5 \\
\hline Saturated (Sat 4) & $(18)$ & 5 \\
\hline Saturated (Sat 5) & $(19)$ & 5 \\
\hline
\end{tabular}

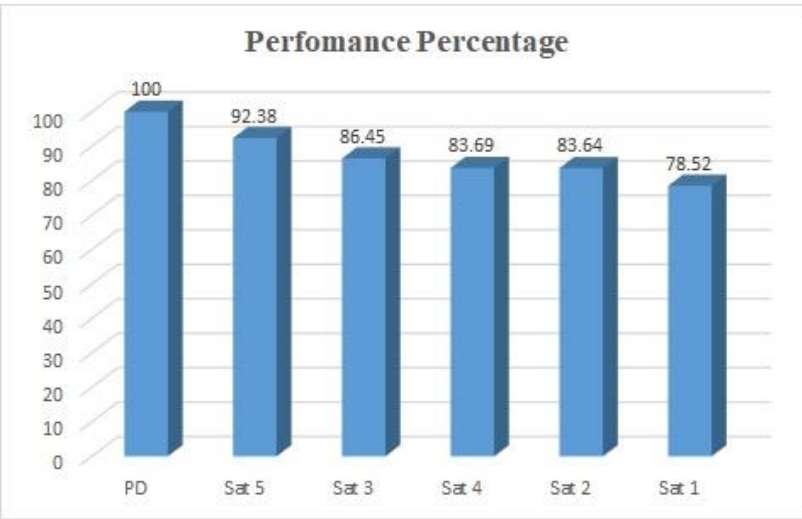

Fig. 16. Performance indexes of evaluated controllers.

In Fig. 16, the performance indexes are shown. The PD control is the highest $\mathcal{L}_{2}$ norm then, the corresponding performance percentage assigned is $100 \%$. The Sat 5 has a performance improvement approximately to $7.3 \%$, later it is improved by Sat 3 roughly $6 \%$ and $13.5 \%$, with respect to PD. The performance of Sat 4 and Sat 5 are very similar and do not have important differences with Sat 3. On other hand, the Sat 1 control is the best performance evaluated improving to $\mathrm{PD}$ regulator approximately to $25.5 \%$. Furthermore, the saturated global controllers with a gamma of variable gains are a solution to regulation problem in joint space, globally asymptotic stability using a strict Lyapunov function is proved, the complicated tune control gains by gamma of variable gains with self-tuning law design is achieved, the servomotor saturation problem is solved, and we have highperformance of regulation using this proposal.

\section{Remarks}

From the above simulation results we highlight the following.

1. The presented cases of saturated schemes with variable gains, have different qualitative proprieties; fast permanent regimen, few oscillations in transitory regime or increase in maximum peak. Each characterize depends on the choice of $\alpha$ and the case of variable gains. It is important to consider according to motion applications on robot manipulators.

2. The optimal value of $\delta_{i}$ is related to the corresponding maximum torque of servomotor. The proposal interval has as its main objective that, the $90 \%$ percent of torque is not exceeded. Hence, incorrect election of $\delta_{i}$ can induces the above scenario. The suggestion is to choose small values of $\delta_{i}$ for small applied torques and big values to big applied torques, without living the interval.

\section{CONCLUSIONS}

According to direct method of Lyapunov, the global regulation is insured. Thus, the saturated controls with variable gains helps to generate an asymptotically stable attractor in overall close-loop system and immunity to initial condition within. When the variable gains are modified by functions of position error and velocity of robot manipulator, the classical energy shaping methodology has been restructured with favorable results, because it was possible to prove the convergence asymptotic of state variables to zero. 
The self-tuning law has the advantage of being easy to implement, because only one parameter is proposed for each degree-of-freedom. Consequently, this represents a simple methodology to any robotics user giving accessibility to experts or beginners. The simulation results obtained, allows to conclude that our propose represents an interesting alternative to implement on industrial robot manipulators of more degrees-freedom and extrapolating to motion control applications such that, control point to point and trajectory tracking, without manual tuning of control gains and saturation of robot actuators. On the other hand, to future works, we want to implement on an experimental directdrive-robot manipulator. Thus, new data can be obtained to complement our proposal.

\section{ACKNOWLEDGMENT}

B. Sánchez-García thanks to the scholarship No. 1008015 granted by CONACyT for studies of master.

\section{REFERENCES}

[1] B. Sánchez-García, E. Rodríguez-Hernández, F. Reyes-Cortés, B. AlHadithi and O. Felix-Beltrán, "Desing and implementation of saturated regulators with variable gains for robot manipulators", Devolvement with mechatronics fucus, Cap. 9, vol. 1, pp. 113-130, 2020.

[2] F. Reyes-Cortes, Robotics: Control of robot manipulators, 1st ed., vol. 1. Grupo editor Alfa Omega, 2011, pp. 331-335.

[3] R. Kelly, V. Santibáñez and F. Reyes-Cortés, "On a saturatedproportional derivative feedback with adaptive gravity compensation of robot manipulators," International Journal of Adaptive Control and Signal Processing, vol. 10, pp. 465-479, 1996.

[4] V. Santibáñez and R. Kelly, "Global regulation for robot manipulators under SP-SD feedback", IEEE International Conference on Robotics and Automation, vol. 1, pp. 927-932, 1996.

[5] V. Santibáñez, R. Kelly and F. Reyes-Cortés, "A new set-point controller with bounded torques for robot manipulators", IEEE Transactions on Industrial Electronics, vol. 45, no. 1, 1998.

[6] D. Lopéz-Araujo, A. Zavala-Rio, V. Santibáñez and F. Reyes-Cortés," An SP-SD-type global adaptive tracking for robot manipulators with bounded torques", XV1 Congreso latinoamericano de Control Automático, pp. 791-797, 2014.

[7] V. Santibáñez and R. Kelly, "A class of nonlinear PID global regulators for robot manipulators", IEEE International Conference on Robotics and Automation, vol 4, pp. 3601-3606, 1998.

[8] D. Sun, S. Hu, X. Shao and C. Liu, "Global stability of a saturated nonlinear PID controller for robot manipulators" IEEE Transactions on Control Systems Technology, vol. 17, no. 4, pp. 892-899, 2009.

[9] M. Takegaki and S. Arimoto, "A new feedback method for dynamic control of manipulators", ASME Journal Dynamic System Measuring Control (103), pp. 119-125, 1981.

[10] F. Reyes-Cortés, J. Barahona and L. Espinosa, "Trigonometric saturated controller for robot manipulators", 2nd WSEAS International Conference on Instrumentation Measurement Control Circuits and Systems, pp. 356-362, 2002.

[11] F. Reyes-Cortés, L. Espinosa and J. Barahona, "Family of saturated controllers for robot manipulators", IASTED International Conference on Control and Applications, pp. 250-255, 2002.

[12] F. Reyes-Cortés, J. Cid, M. A. Limón and M. Cervantes, "Square roottype control for robot manipulators", International Journal of Advance Robotic Systems, vol. 10, no. 39, pp. 1-7, 2013.

[13] F. Reyes-Cortés, O. Felix-Beltrán, J. Cid and G. Alonso, "A family of hyperbolic-type control schemes for robot manipulators", Kybernetika, vol. 55, no. 3, pp. 561-585, 2019.
[14] R. Kelly, R. Haber, R. Haber-Guerra and F. Reyes-Cortés, "Lyapunov stable control of robot manipulators: A fuzzy self-tuning procedure", Intelligent Automation and Soft Computing, vol. 5, no.4, pp. 313-326, 1999.

[15] M. A. Llama, R. Kelly and V. Santibáñez, "A stable motion control system for robot manipulators via fuzzy self-tuning", Fuzzy Sets and Systems, Elsevier, vol. 124, pp. 133-154, 2001.

[16] J. L. Meza, V. Santibáñez, R. Soto and M. A. Llama, "Fuzzy selftuning PID semiglobal regulator for robot manipulator", IEEE Transactions on Industrial Electronics, vol 59, no. 6, pp. 2709-2717, 2012.

[17] M. Van, X. Do and M. Mavrovouniotis," Self-tuning fuzzy PIDnonsingular fast terminal sliding mode control for robust fault tolerant control of robot manipulators", ISA Transactions, Elsevier, no. 96, pp. 60-68, 2019.

[18] K. Zhang and X. An, "Design of multivariable self-tuning PID controllers via quasi-diagonal recurrent wavelet neural network", Second International Conference on Intelligent Human-Machine Systems and Cybernetics, pp. 95-99, 2010.

[19] S. Al-Khayyt, "Tuning PID Controller by neural network for robot manipulators trajectory tracking", Al-khwarizmi Engineering Journal, 2013, vol. 9, no.1, pp. 19-28, 2013.

[20] N.Jaisumroum, P.Chotiprayanakul and S. Limnararat, "Self-tuning control with neural network for robot manipulator", $16^{\text {th }}$ International Conference on Control Automation and Systems (ICCAS), pp. 10731076, 2016

[21] H. Nohooji, "Constrained neural adaptive PID control for robot manipulators", Journal of the Franklin Institute, Elsevier, vol. 357, no. 7, pp. 3907-3923, 2020.

[22] F. Reyes-Cortés and B. Al-Hadithi, "An asinh-type regulator for robot manipulators with global asymptotic stability", Automatika, vol.61, no.4, pp. 574-586, 2020.

[23] R. Kelly and R. Carelli, "A class a of nonlinear PD-type controllers for robot manipulators", Journal of Robotic Systems, vol. 13, no 12, pp.793-802, 1996

[24] F. Reyes-Cortés, J. Cid, and C. Campuzano, "Development of an experimental platform with open architecture for robot manipulators", IAESTED International Conference Modeling and Simulation, pp. 7781, 1999.

[25] F. Salas, V. Santibáñez and M. A. Llama, "Variable gains PD tracking control for robot manipulators: Satability analysis and simulation", World Automation Congress, IEEE, pp. 1-6, 2012.

[26] J. Sifuentes, J. L. Meza, "A global asymptotic stable quasi variable PID regulator for robot manipulators", Congreso Nacional de Control Automático, pp. 233-238, 2012.

[27] O. Ramos, F. Reyes-Cortés, M. A. Vargas and S. Vergara, "Variable control gains for PD controller for robot manipulators", 2015 International, Conference on Mechatronics Electronics and Automotive Engineering, pp- 187-192, 2015.

[28] M. A. Limón-Diaz, F. Reyes-Cortés and E. González-Galván, "Unbounded regulators with variable gains for a direct-drive robot manipulator", Automaika: Journal for control Mensurament Electronics Computing and Communications, Kprema IFAC, vol. 57, no.2, pp. 484-495, 2016.

[29] M. A. Limón-Diaz, F. Reyes-Cortés and E. González-Galván, "Saturated regulation with derivative gain for robot manipulators", Revista Iberoamericana de Automática e Informática Industrial, RIAI Elsevier, no. 14, pp. 434-445, 2017.

[30] F. Reyes-Cortés and R. Kelly, "Experimental evaluation of modelbased controllers on a direct-drive robot arm", Mechatronics 11, 2000 Elsevier Science, pp. 267-282, 2001.

[31] R. Kelly, V. S. Davila, and A. Loria, "Control of robot manipulators in joint space", Advanced Textbooks in Control and Signal Processing, 2005. 